\title{
ANÁLISE DA PRODUÇÃO CIENTÍFICA NO CAMPO DE ESTUDO DAS REDES EM PERIÓDICOS NACIONAIS E INTERNACIONAIS
}

\author{
Fabiela Fatima Andrighi \\ Mestre em Turismo e Hotelaria pela Universidade do Vale do Itajaí - UNIVALI \\ Professora da Universidade do Vale do Itajaí - UNIVALI \\ fabiela@univali.br
}

\section{Valmir Emil Hoffmann}

Doutorado em Administração de empresas pela Universidad de Zaragoza, Espanha

Professora Universidade de Brasília - UNB

ehoffmann@univali.br

\section{Marcos Antonio Ribeiro Andrade}

Mestre em Administração pela Universidade do Vale do Itajaí - UNIVALI

Professor do Centro Universitário de Volta Redonda - UNIVALI

mandrade@univali.br

\section{RESUMO}

Este estudo teve como objetivo mensurar a produção de pesquisas de redes, através do produto final das pesquisas - as publicações científicas. Diversas abordagens teóricas sobre o tema redes oferecem potencial diálogo entre os vários ramos das ciências sociais aplicadas. Autores contemporâneos afirmam que as empresas em busca de competitividade, encontraram na formação de redes uma alternativa estratégica. Desde os primeiros trabalhos nos anos 1980, o conhecimento sobre o tema cresceu no Brasil e no mundo. Expandindo-se de maneira não linear, o estado da arte se ampliou sem, contudo, dar uma resposta uníssona sobre questões simples, até mesmo sobre o conceito de redes. Levando em conta o escopo deste trabalho, optou-se pela abordagem do tipo quantitativa, por meio de uma pesquisa documental, utilizando-se a bibliometria, aplicada em periódicos nacionais e internacionais (Qualis A), que concentraram a publicação em estratégia, no período de 2000 a 2005, na área de Ciências da Administração. Os resultados apontam a predominância do termo redes nos estudos nacionais e internacionais; cerca de $30 \%$ dos periódicos pesquisados publicaram aproximadamente $45 \%$ dos artigos encontrados sobre o tema; a quantidade de publicações em 2000 estava em um patamar superior ao de 2002-2003, mas que voltou a crescer em 2005; e, por fim, há diferenças entre os estudos realizados no Brasil e aqueles efetuados no exterior.

Palavras-chave: Redes; Relações interorganizacionais; Aglomerações; Bibliometria. 


\section{INTRODUÇÃO}

As preocupações atuais sobre competitividade não são recentes. No Brasil, a decisão de abertura de mercado no início dos anos 1990 ensejou novas perspectivas ao país, em termos de acesso a mercados. Naquele período, Lewis (1992) destacava que o mundo estava claramente se tornando um mercado único, elevando os padrões para as empresas alcançarem o sucesso competitivo e esse parece ser um processo ainda em andamento. Esse mercado único dinamizou as empresas, e a busca pelo incremento da competitividade individual e coletiva constituiu seu objetivo constante e também o dos governos, e fator crítico para a prosperidade das companhias, das cidades, das regiões e dos países, inseridos em um ambiente de concorrência global (Amato Neto, 2005).

Ser competitivo significa dispor de vantagem competitiva sustentável, o que para Coyne (1986) ocorre quando o produto/serviço da empresa (cidade, região ou país) é preferido pelo consumidor ao longo do tempo, em detrimento daquele ofertado pelos concorrentes. Para compreensão da vantagem competitiva e da adequação à dinâmica da competição, almejam-se soluções para organizações que pretendem uma gestão por meio de novos modelos organizacionais - as redes. Essas estruturas empresariais possibilitam, em alguns casos, que concorrentes se tornem potenciais parceiros através de ações de cooperação (Begnis, Pedrozo, \& Estivalete, 2005), na busca por alternativas para a resolução de problemas.

As redes passaram a ser consideradas como uma opção estratégica para competitividade setorial. Essa nova forma de organização das empresas, segundo pesquisadores como Castells (1999) e Jarillo (1988), entre outros, teve início na década de 1980, ganhando impulso a partir do final do século XX e início do século XXI (Hoffmann, Molina-Morales, \& Martinez-Fernádez, 2007), fundamentada nos benefícios gerados pela aglomeração territorial e/ou pela cooperação entre firmas. Comumente, a literatura expressa as relações interorganizacionais, principalmente por comportamentos de competição, cooperação, confiança. Begnis et al. (2005) evidenciam a cooperação e a parceria como base nos relacionamentos interfirmas, que originam os arranjos interorganizacionais, com objetivo final de alcançar um âmbito diferenciado de competitividade: a interorganizacional. Nesse contexto, o meio acadêmico respondeu inicialmente com vários trabalhos, que destacaram as vantagens dos diferentes tipos de relacionamentos interorganizacionais (Begnis et al., 2005).

Decorridos mais de vinte anos da publicação dos primeiros trabalhos, pode-se dizer que houve avanços. No entanto, como a polissemia é característica desse tema (Jarillo, 1988; Powell, 1990; Ring; Van de Ven, 1992), parece relevante compreender como ele foi tratado nas publicações em periódicos nacionais e internacionais. Nesse sentido, este trabalho teve por objetivo mensurar a produção de 
pesquisas de redes em periódicos nacionais e internacionais, indicadas pelo portal CAPES, com conceito "A" (Qualis A), até dezembro de 2005. Estudos anteriores sobre esse tema foram desenvolvidos por Oliver e Ebers (1998), entre outros no âmbito internacional, que foram replicados no Brasil por Balestrin et al. (2010) e Balestrin e Verschoore (2008). Um trabalho anterior executado por Hoffmann et al. (2007) também utilizou a pesquisa bibliométrica em periódicos no período de 1996 a 2004. Todavia, esse último trabalho focou em artigos nacionais que abordavam o termo Redes de Empresas. Parece importante conhecer o padrão de crescimento das publicações sobre redes e a sua distribuição entre os periódicos, para visualizar a intensidade, aspectos metodológicos e conceituais a partir dos quais o tema está sendo discutido no Brasil e no exterior, como forma de contribuir para futuras pesquisas.

Para a consecução do objetivo desta investigação, lançou-se mão de um recurso que começa a despertar o interesse dos estudiosos, quando se trata de um levantamento do estado da arte, com enfoque especial no quantumm - a bibliometria. A bibliometria tem servido à comunidade científica como ferramenta estatística para a gestão da informação e do conhecimento, além de constituir-se um instrumento de avaliação científica e tecnológica. De acordo com Guedes e Borschiver (2007), ela permite mapear e gerar diferentes indicadores de tratamento.

Segundo Hoffman e Hoolbrook (1993), foi Price, em 1976, quem cunhou a expressão bibliometria para designar o estudo formal das citações, principalmente para investigar as comunidades científicas. Contudo, esse pioneirismo não é consenso. Para Culnan (1986), um dos antecessores foi Pritchard em finais dos anos 1960. Segundo a autora, o conceito envolve a contagem de citações de publicações, em um campo da literatura científica e o uso dessa contagem para se fazer uma distribuição estatística. Sua utilidade reside na aceitação que as publicações anteriores podem ter em um projeto, ou seja, as citações são uma medida de dependência em trabalhos anteriores, complementa Culnan (1986).

Apesar da polêmica acerca dos primórdios do conceito, percebe-se que como estratégia de pesquisa a bibliometria está presente e seu interesse tem se incrementado. Em um sistema de busca de publicações científicas (EBSCO®), com esse tema somado à administração, os 114 trabalhos disponíveis vão de 1984 a 2008, sendo a metade deles entre 2005 e 2008. A justificativa pelo crescente interesse talvez seja, como já afirmava Pasadeos (1985), a necessidade, ainda atual, de se saber quem citar e quais são os meios mais utilizados para consulta, ao se elaborar ou executar uma pesquisa. Uma das repercussões das citações, escrevem Chung e Cox (1990, p. 301), é que o "sucesso alimenta o sucesso", ou seja, um artigo frequentemente citado no passado tem maior probabilidade de ser citado no futuro que outro pouco citado.

Revista de Administração e Inovação, São Paulo, v. 8, n. 2, p.29-54, abr./jun. 2011 
$\mathrm{O}$ artigo se estrutura, além desta introdução, em outros cinco tópicos. No primeiro há uma revisão bibliográfica seguida de algumas notas sobre bibliometria; e logo após são evidenciados os aspectos metodológicos empregados para o seu desenvolvimento. Em seguida são apresentados os resultados e análise, numa visão panorâmica da produção acadêmica com suas abordagens e, nas considerações finais, promove-se uma breve discussão dos resultados com base nas referências conceituais utilizadas.

\section{FUNDAMENTAÇÃO TEÓRICA}

No contexto internacional, os autores Jarillo (1988), Miles e Snow (1986), Powell (1990) e Thorelli (1986) e no Brasil, Amato Neto (2000), Casarotto Filho e Pires (1999) e Siqueira (2000), encontram-se entre as primeiras referências a discutir a temática de redes. Posteriormente, outros estudos ampliaram a pesquisa no Brasil, como os trabalhos de Carrão (2004), Pitassi e Macedo-Soares (2003), Teixeira e Guerra (2002) e como indicam Hoffmann et al. (2007).

Jarillo (1988) sustenta que, não obstante a dificuldade conceitual, os artigos acerca do tema, principalmente aqueles oriundos da Europa, e as ideias que apontam, os habilitam como blocos construtores em uma teoria de redes estratégicas. Com relação à definição de redes, para Thorelli (1986), elas são posições ocupadas pelas empresas e as ligações manifestadas por essas posições. Diferentemente, Jarillo (1988, p.32) entende que as redes, que ele chamou de estratégicas, "são acordos de longo prazo entre empresas lucrativas distintas que as permite obter uma vantagem competitiva frente aos competidores de fora da rede". As relações desenvolvidas pelas empresas dentro das redes são essenciais para sua posição competitiva, complementa o autor.

As abordagens - e efetivamente o conceito de rede empregado - variam conforme o instrumental analítico e as bases teóricas de cada área, que são muitas vezes difusos e apresentam diferentes taxonomias relacionadas às especificidades dos distintos programas de pesquisa e de seus objetivos (Cassiolato \& Szapiro, 2003). Apesar disso, os trabalhos sobre redes ganharam um caráter interdisciplinar, ancorados em perspectivas tanto relacionadas à Teoria das Organizações como também a abordagens ligadas à estratégia. A ideia que permeia a maioria das investigações sobre redes nas Ciências Sociais Aplicadas a aponta como uma forma alternativa de organização do modelo produtivo. Ou seja, como escreveu Powell (1990), uma possibilidade distinta de organização que não se configura como hierarquia, aquela em que a empresa detém total controle do processo e as relações se baseiam em poder de comando, nem como mercado, no qual as relações são caracterizadas pela

Revista de Administração e Inovação, São Paulo, v. 8, n. 1, p.29-54, abr./jun. 2011 
barganha e pelo mecanismo de preços. Dessa maneira, a rede é uma forma de organização singular e que não se confunde com as duas formas anteriores (hierarquia e mercado).

Em estudos do sociólogo e pesquisador Castells (1999), os conceitos de competitividade em relação às redes de empresas são elementos associados ao paradigma organizacional, que atua paralelamente com as ferramentas tecnológicas, o que possibilita às empresas alcançarem uma vantagem competitiva no mercado, difícil de ser obtida se essa atuação for realizada de maneira isolada, via hierarquia, por exemplo.

O termo redes de empresas pode ser encontrado sob diferentes formas, em distintos contextos e com semânticas diversas, o que cria a polissemia já assinalada (clusters, arranjos produtivos locais, distrito industrial, redes de empresas e redes interorganizacionais), como se observa nos estudos de Castells (1999). O autor faz referência às "redes familiares chinesas e no norte da Itália até as redes internacionais resultantes de alianças estratégicas entre empresas" (Castells, 1999, p. 214). Para Amato Neto (2000), as redes são consideradas um agrupamento de empresas cujo objetivo principal é o fortalecimento das atividades de cada membro e completa-se tanto nos aspectos técnicos como administrativos, e o relacionamento decorrente pode ter prazo indeterminado e ser aberto para novos participantes. As redes são articulações de "cooperação e colaboração entre empresas", conforme Hall (2001, p. 233).

Retomando os autores seminais, no conceito de Thorelli (1986), uma rede de empresas é o que há de intermediário entre uma simples empresa e o mercado e segundo Miles e Snow (1986) com combinação única de estratégias, estrutura e processo de gestão ao qual se refere. Para efeito deste estudo, determina-se como conceito operacional aquele formulado por Jarillo (1988), para quem as redes são acordos de longo prazo que definem objetivos comuns para a obtenção de vantagens competitivas perante os concorrentes, ressalvando-se apenas o fato de aqui se considerar que esses acordos podem ser formais ou não, tal como escreveram Hoffmann et al. (2007).

Em termos de propósito, para Miles e Snow (1986), o estabelecimento de redes se dá como resposta às complexas e mutantes condições competitivas. Como afirmam Balestrin e Verschoore (2008), as redes interorganizacionais visam reunir em uma única estrutura as características essenciais ao novo ambiente competitivo, sustentada por uma governança altamente descentralizada, o que evita que as empresas envolvidas percam a flexibilidade e a agilidade. Levando-se em conta a cronologia entre esses dois textos, com uma diferença de mais de vinte anos, percebe-se que as condições competitivas são as mesmas, ou seja, intensa competição, e a resposta a esse cenário também é a mesma, o estabelecimento das redes.

Revista de Administração e Inovação, São Paulo, v. 8, n. 2, p.29-54, abr./jun. 2011 
Parece que cabe ressaltar que o termo redes está comumente relacionado aos comportamentos de cooperação, comunicação e confiança. A cooperação é um comportamento, que pode realizar transformações importantes nas organizações participantes, que muda as percepções sobre competição e possibilita considerar os concorrentes como parceiros (Powell, 1990), além de ser foco para o desenvolvimento da competitividade interorganizacional (Verschoore Filho, 2003).

A confiança pode ser outro comportamento perceptível para a formação de redes interorganizacionais (Amato Neto, 2000; Hall, 2001; Jarillo, 1988; Powell, 1990;), pois permite economia de custos de transação, em virtude de dispensar o uso de contratos nas relações (Williamson, 1991), o que pode viabilizar também a cooperação. Neste tema, a aglomeração enseja relações próximas que favorecem o estabelecimento de confiança, de troca de informações detalhadas e resolução conjunta de problemas (Nakano, 2005).

Outro comportamento determinante na capacidade de articulação dos componentes das redes interorganizacionais e, consequentemente, para o seu desempenho é a comunicação (Castells, 1999). Relacionamento com base em confiança, troca de informações (comunicação) e ações coletivas, são aspectos que normalmente estão imbricados à cooperação interorganizacional (Knorringa \& MeyerStamer, 1999).

\section{ALGUMAS NOTAS SOBRE O USO DA BIBLIOMETRIA}

A pesquisa bibliométrica tem sido empregada com diversos fins. Mahon e Andrews (1987) estudaram 375 artigos nos principais periódicos apontados por um grupo de pesquisadores através de questionário. Suas inquietações os levaram a determinar quais os trabalhos e os periódicos mais citados e se as citações presentes nos artigos estavam aumentando de número. Chun e Cox (1990) estudaram 15 periódicos na área de finanças, atingindo um total de 6.270 autores. Nesse universo, com uma frequência de $62,2 \%$ apareceram aqueles que haviam publicado apenas uma vez. Os autores repetiram o estudo em contabilidade e chegaram a resultados semelhantes (Chung, Pak \& Cox, 1992).

O trabalho de Hoffman e Hoolbrook (1993) procurou determinar a frequência de publicação por autor, de citações e por revista. McMillan e Hamilton III (2000) estudaram os trabalhos apresentados pelas empresas na indústria farmacêutica, como uma medida do conhecimento organizacional e utilizaram a estatística descritiva para apresentar os resultados.

Mais recentemente, McMillan e Casey (2007) investigaram as bases teóricas empregadas em artigos de um periódico e descobriram que elas se alteraram ao longo do tempo. O'Leary (2007) usou

Revista de Administração e Inovação, São Paulo, v. 8, n. 1, p.29-54, abr./jun. 2011 
o índice de citações sociais para determinar os artigos mais citados em um periódico nos seus 25 volumes e o resultado apontou que a maioria dos autores aparece apenas uma vez. Carter, Leuschner e Roger (2007) pesquisaram um periódico com 40 anos de publicação para identificar as fontes utilizadas, nas quais foram baseadas as pesquisa presentes no periódico. Eles utilizaram as redes sociais para entender os relacionamentos entre os autores.

No Brasil vários trabalhos recentes aplicaram a bibliometria como enfoques em alguns casos semelhantes àqueles da literatura internacional, e outros inovadores. Mendonça Neto, Riccio e Sakata (2006) analisaram a distribuição, as características metodológicas, a evolução, a temática, e a produtividade da publicação em contabilidade. Em outro trabalho, Mendonça Neto et al. (2006) analisaram a evolução temporal da produção da pesquisa científica em contabilidade no Brasil no período de 1981 a 2005. Para apresentar os resultados, os autores utilizaram frequência absoluta e figuras.

Com a proposta de levantar, caracterizar e analisar as fontes de informação utilizadas na construção de teses de doutorado em contabilidade, Magalhães (2006) desenvolveu seu trabalho tendo como objeto as referências utilizadas, e aplicou a estatística descritiva. Na mesma linha do trabalho de Magalhães (2006), Chan, Milani Filho e Martins (2007) analisaram a relação entre as áreas temáticas e a natureza das plataformas teóricas em um congresso de contabilidade. Através de uma análise por correspondência, mostraram que há áreas que usam mais periódicos e anais, outras que usam mais livros e outras ainda que utilizam mais literatura técnica. Rossoni et al. (2008) analisaram os padrões de cooperação entre pesquisadores da área de administração da informação. Para isso utilizaram as publicações preliminares realizadas nos anais do Encontro da Associação Nacional de Pós-Graduação e Pesquisa em Administração - ENANPAD -, entre 2002 e 2006. O estudo das metodologias utilizadas também foi objeto de uma pesquisa bibliométrica realizada por Gallon et al. (2007), em periódicos científicos classificados com A-Nacional pelo sistema Qualis, entre 2000 e 2006. Para a área, os autores ainda contribuem no sentido de indicar as obras mais citadas e a tendência dos temas mais comuns.

Moretti e Figueiredo (2007) realizaram uma investigação com o objetivo de demonstrar que existe uma reprodução de discurso de uns poucos autores, no campo da responsabilidade social empresarial. Eles usaram a estatística descritiva e a análise de correspondência para apresentar seus dados. Gallon e Cunha (2007) apresentaram as estratégias metodológicas dos estudos etnográficos publicados no ENAMPAD 2000-2006. Os autores classificaram seu trabalho como exploratório e descritivo. 
Com o intuito de analisar a utilização da Análise Envoltória de Dados (DEA), Macedo, Nova e Almeida (2007) pesquisaram as publicações em eventos e periódicos brasileiros Qualis A. Os autores relatam o número de artigos por ano e indicam o local de publicação.

Tratando-se de redes, um estudo considerado seminal no uso da bibliometria foi realizado por Oliver e Ebers (1998). O objetivo do trabalho foi analisar o campo de estudo do tema redes, através da pesquisa de 158 artigos publicados em quatro periódicos internacionais. Os autores identificaram quatro perspectivas substantivas de pesquisa: redes sociais, poder e controle, institucional, economia institucional e estratégia de clusters. Eles também demonstraram as áreas periféricas, as principais, as já superadas e outras em consolidação. A pesquisa apontou que a publicação cresceu, principalmente entre 1990 e 1996. As teorias mais utilizadas segundo os autores foram a dependência de recursos, de redes, institucional, de poder político e de estratégia. Além disso, Oliver e Ebers (1998) deram uma contribuição no sentido de esclarecer os antecedentes teóricos utilizados.

Nessa mesma linha foram apresentados dois trabalhos no Encontro da Divisão de Estudos Organizacionais (ENEO) da ANPAD 2008, no subtema de redes e relações inter-organizacionais. O primeiro deles, de autoria de Balestrin e Verschoore (2008), que foi publicado em revista posteriormente, Balestrin et al. (2010) tratou de reproduzir o estudo de Oliver e Ebers (1998), no Brasil, através de periódicos Qualis A nacional na área de administração e uma publicação de evento do ENANPAD no período de 2000 a 2006. Outro trabalho que seguiu a pesquisa de Oliver e Ebers (1998) foi apresentado por Puffal e Tondolo (2008). O estudo analisou a configuração conceitual dos trabalhos sobre as relações interorganizacionais publicados em periódicos internacionais de 1997 a 2007. A investigação identificou 42 artigos nos mesmos periódicos que haviam sido pesquisados por Oliver e Ebers (1998). Os resultados mostram que 95,2\% dos artigos eram estudos empíricos e os demais estudos teóricos; 54,8\% possuíam abordagem qualitativa, 21,4\% qualitativa e quantitativa e $19 \%$ somente quantitativa. A técnica qualitativa mais utilizada foi o estudo de caso $(23,8 \%)$ e a de levantamentos, a quantitativa mais frequente $(47,6 \%)$. As abordagens teóricas mais presentes foram as redes contingência, institucional, dependência de recursos, política e poder.

\section{METODOLOGIA}

Este trabalho assume um caráter exploratório, uma vez que busca compreender o campo de estudo de redes e com isso se pode indicar direcionamentos em termos de investigações futuras no

Revista de Administração e Inovação, São Paulo, v. 8, n. 1, p.29-54, abr./jun. 2011 
tema. A pesquisa foi desenvolvida entre 2006 e início de 2007, e como algumas publicações escolhidas não estavam atualizadas, para evitar certo viés, decidiu-se pelo período de 2000 a 2005.

A abordagem adotada para a realização deste trabalho foi quantitativa, através de uma pesquisa documental utilizando a bibliometria. A escolha pelos periódicos classificados pela Coordenação de Aperfeiçoamento de Pessoal de Nível Superior (CAPES) se deu pelo fato de que essa listagem de periódicos segue parâmetros de indexação das revistas e pelo fator de impacto. Pelo grau de importância do periódico, decidiu-se, no caso dos periódicos nacionais, pelos que possuíam o conceito "A" em 2005, ano-base da coleta (Qualis A-nacional), que então estavam entre os de alto impacto.

Dessa forma, foram utilizados como bases da pesquisa os periódicos: Revista Organizações e Sociedade (O\&S); Revista de Administração Contemporânea (RAC); Revista de Administração de Empresas (RAE); Revista de Administração Pública (RAP); Revista de Administração da Universidade de São Paulo (RAUSP); Revista Eletrônica de Administração (REAd) e a Revista de Estudos Econômicos (EE), que também publica artigos da área de Administração dentro do tema de redes interorganizacionais. Note-se que essas revistas concentram a maior parte da publicação em estratégia. Essas fontes também serviram de base para outros trabalhos no Brasil, como os de Balestrin et al. (2010); Balestrin e Verschoore (2008); Gallon et al. (2007); Macedo et al. (2007); e Mendonça Neto et al. (2006) e, o que reforçou a escolha. Para seleção do artigo, utilizou-se como critério a presença das palavras redes, alianças, clusters e parcerias no título, e/ou resumo e/ou palavras-chave.

Para os periódicos internacionais, inicialmente realizou-se um levantamento das revistas da área de estratégia organizacional, classificadas em internacional "A" pela CAPES utilizando o buscador Proquest ${ }^{\circledR}$, no qual foram identificados os periódicos disponíveis para a seleção de artigos. Os periódicos investigados foram: Academy of Management Journal (AMJ); Administrative Science Quartely (ASQ); Harvard Business Review (HBR); Industrial Management (IM); Industrial Marketing Management (IMM); Journal of International Business Studies (JIB); Journal of Organization Change Management (JOC); $R \& D$ Managemente (RDM); Research Policy (RP); Strategic Management Journal (SMJ); Technovation (TEC); e World Development (WD). A pesquisa foi executada no título, resumo e palavras-chave a partir das palavras network, alliances clusters e partnerships. 


\begin{tabular}{|c|c|c|c|}
\hline TERMOS & $\begin{array}{c}\text { ABORDAGEM DA } \\
\text { PESQUISA }\end{array}$ & $\begin{array}{c}\text { TIPO DE } \\
\text { PESQUISA }\end{array}$ & CONSTRUTOS \\
\hline Redes & Quantitativa & Descritiva & Competitividade \\
\hline aliança & Qualitativa & Exploratória & Cooperação \\
\hline clusters & & Ensaio teórico & Confiança \\
\hline & & & Conhecimento \\
\hline & & & Aprendizagem \\
\hline
\end{tabular}

Quadro 1: Categorias de pesquisa.

Fonte: Elaborado pelos autores.

Foram pesquisadas as categorias apresentadas no Quadro 1. A opção pela categoria termos assemelha-se àquela realizada por Carter et al. (2007), e tem o objetivo próximo ao do trabalho apresentado por Gallon et al. (2007). Como são construtos distintos, mas estão relacionados, sua incidência pode traçar um parâmetro de hegemonia de termos. Tanto a abordagem da pesquisa como o tipo esquadrinham a possibilidade de orientação com relação à metodologia da pesquisa. Ou seja, pode ser um indicativo de metodologias aceitáveis, por seu uso, ou ainda daquelas que podem ter um caráter experimental, que assim podem ser orientadoras de investigações mais avançadas. A opção pelo estudo das metodologias de pesquisa também já foi utilizada por Gallon et al. (2007) e Gallon e Cunha (2007).

O tratamento dos dados foi realizado com estatística descritiva e por análise de correspondência. Os trabalhos de Chung e Cox (1990); Hoffman e Hoolbrook (1993); Mahon e Andrews (1987); Oliver e Ebers (1998) no exterior; e Balestrin et al. (2010); Macedo et al. (2007); Moretti e Figueiredo (2007); Puffal e Tondolo (2008) no Brasil são algumas das fontes que previamente utilizaram a estatística descritiva para apresentar seus resultados, sobretudo a frequência absoluta, que é empregada neste trabalho. Como um recurso auxiliar e baseado no trabalho de Pasadeos (1985), igualmente apresentam-se gráficos, para se poder visualizar os resultados nas categorias.

Os trabalhos anteriores de Hoffman e Hoolbrook (1993) e Kostoff et al. (2007), no exterior, e de Chan et al. (2007) e Moretti e Figueiredo (2007), no Brasil, despertaram o interesse nestes autores para a possibilidade de estabelecer uma análise de correspondência, a partir das dimensões termos que englobou os termos e construtos e aspectos metodológicos que abarcaram a abordagem de pesquisa e o tipo de pesquisa, detalhados no Quadro 1. 


\section{RESULTADOS}

Conforme já indicado na metodologia, foram selecionados 40 artigos em sete revistas Qualis "A" de circulação nacional. A Tabela 1 mostra um incremento no número de publicações nos anos pesquisados; $40 \%$ dos trabalhos foram encontrados nos anos de 2004 e 2005 . Exceção é feita ao ano de 2000 com 20\% das publicações do período pesquisado, sendo nesse ano 62,5\% deles decorrentes da Revista de Administração Pública (RAP). A partir de 2002, observa-se expansão, e os anos de 2002 a 2005 totalizaram 70\% de todos os artigos. Essa sinalização denota que no Brasil o tema continua "de moda", mais de dez anos depois de Jarillo (1988) ter usado essa expressão para referir-se à pesquisa internacional.

Tabela 1 - Número de artigos selecionados por periódico Qualis "A" de circulação nacional entre os anos de 2000 e 2005.

\begin{tabular}{cccccccccc}
\hline Ano & EE & O\&S & RAC & RAE & RAP & RAUSP & REAd & Total & $\%$ \\
\hline 2000 & 1 & - & - & 1 & 5 & - & 1 & 8 & 20 \\
\hline 2001 & - & 1 & 1 & - & 1 & 1 & - & 4 & 10 \\
\hline 2002 & 1 & - & - & 2 & - & 2 & 1 & 6 & 15 \\
\hline 2003 & - & - & 1 & 1 & 1 & 1 & 2 & 6 & 15 \\
\hline 2004 & - & - & 3 & 3 & 1 & - & - & 7 & 17,5 \\
\hline 2005 & 1 & 2 & 1 & 1 & 2 & 1 & 1 & 9 & 22,5 \\
\hline Total & 3 & 3 & 6 & 8 & 10 & 5 & 5 & 40 & 100 \\
\hline$\%$ & 7,5 & 7,5 & $\mathbf{1 5}$ & $\mathbf{2 0}$ & $\mathbf{2 5}$ & 12,5 & 12,5 & 100 & \\
\hline
\end{tabular}

Fonte: Elaborado pelos autores.

Observa-se que do total de artigos encontrados, $45 \%$ foram publicados na Revista de Administração de Empresas (RAE) e Revista de Administração Pública (RAP) - 18 artigos -, seguidas da Revista de Administração Contemporânea (RAC) e Revista de Administração da Universidade de São Paulo (RAUSP). Pondera-se que os estudos sobre o tema redes têm maior frequência nas publicações de Administração, pois está diretamente ligada ao incremento da competitividade (Jarillo, 1988) e como forma alternativa de organização do modelo produtivo. Ainda sobre esse crescimento, pode-se inferir que ele espelha o interesse no tema, e que continua em ascensão, considerando que em 2006 a RAE publicou uma chamada especial sobre o tema (v. 46, n. 3) e alguns eventos na área de administração elegeram o tema como eixo principal de discussão. 
Os dados sobre a expansão no Brasil parecem acompanhar um movimento que também é percebido no exterior. Dos 140 artigos pesquisados em 12 periódicos (Tabela 2), quase a metade (sessenta e três) são dos anos 2004 e 2005. Do total de trabalhos, destacam-se Academy Management Journal, Administrative Science Quarterly, Strategic Management Journal com 57,8 \% das publicações conforme os critérios estabelecidos. A questão do volume de produção internacional é notória. Esses são periódicos com alto impacto que abarcam a produção de pesquisadores de diversos países. Oliver e Ebers (1998), em sua pesquisa sobre o tema, já haviam sinalizado que o tema havia crescido. Os dados desta pesquisa confirmam que aquela expansão continuou acontecendo internacionalmente, por mais dez anos pelo menos.

Tabela 2 - Número de artigos selecionados por periódico Qualis "A" de circulação internacional entre os anos de 2000 e 2005.

\begin{tabular}{c|c|c|c|c|c|c|c|c|c|c|c|c|c|c}
\hline Ano & AMJ & ASQ & HBR & IM & IMM & JIB & JOC & RDM & RD & SMJ & TEC & WD & Total & $\%$ \\
\hline 2000 & 7 & 6 & - & 3 & 4 & 2 & 3 & 3 & 1 & 10 & 4 & 1 & 44 & 7 \\
\hline 2001 & 1 & 5 & - & 2 & - & - & - & - & 1 & - & 1 & & 10 & 1 \\
\hline 2002 & 6 & 3 & - & - & 1 & - & 1 & 1 & - & - & 1 & 1 & 14 & 6 \\
\hline 2003 & 3 & 4 & - & - & - & - & - & 1 & - & - & 1 & - & 9 & 3 \\
\hline 2004 & 10 & 3 & 2 & 2 & - & 2 & - & - & - & 1 & 3 & - & 23 & 10 \\
\hline 2005 & 8 & 5 & 7 & 3 & - & - & 1 & 1 & - & 9 & 6 & - & 40 & 8 \\
\hline Total & 35 & 26 & 9 & 10 & 5 & 4 & 5 & 6 & 2 & 20 & 16 & 2 & 140 & 35 \\
\hline$\%$ & $\mathbf{2 5 , 0}$ & $\mathbf{1 8 , 5}$ & $\mathbf{6 , 4}$ & 7,2 & 3,6 & 2,8 & 3,6 & 4,4 & 1,4 & 14,3 & 11,4 & 1,4 & 100 & \\
\hline
\end{tabular}

Fonte: Elaborado pelos autores.

No Brasil, pelo que se pode perceber, há uma visão sobre as redes que a discute como um modelo organizacional, mais voltado à adaptação a um contexto competitivo, isto é, ligado à estratégia. No exterior, essa etapa parece ter sido vivenciada até o final do século passado, quando então as preocupações se voltaram mais para responder questões ligadas a aspectos mais organizacionais, propriamente. Um exemplo disso é o trabalho de Dyer (1996) e Echols e Tsai (2005), ambos publicados na Strategic Management Journal. Em Dyer (1996), percebe-se claramente a preocupação do autor em esclarecer os ganhos em termos objetivos, ao discutir vantagem competitiva. Já no trabalho de Echols e Tsai (2005) aborda-se um tema mais organizacional, a saber, a questão do imbricamento, ou arraigo. 
Conforme apresentado na Figura 1, verifica-se que houve uma redução do número de artigos publicados em 2001 constituindo nesse período um valor percentual de 17,14\% e em 2003, 21,43\% do total de artigos publicados nos periódicos nacionais e internacionais. O crescimento foi retomado a partir de 2004. Percebe-se que a redução mais notável foi entre os periódicos internacionais, considerando que nas publicações nacionais entre 2002 e 2004 a variação foi menor.

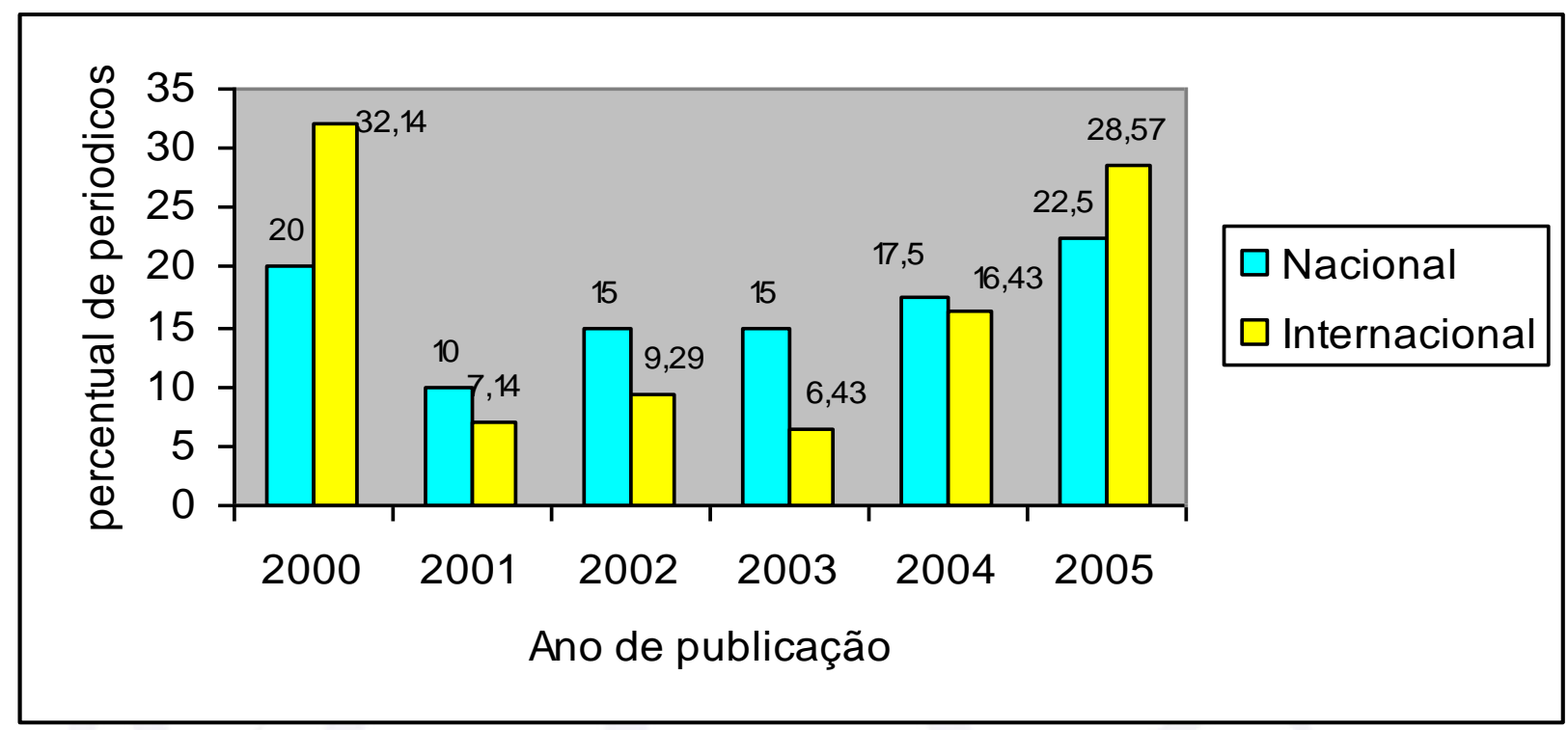

Figura 1: Comparativo de artigos publicados nos periódicos

Fonte: Elaborado pelos autores.

Considerando os termos, observa-se a distribuição nos periódicos nacionais e internacionais (Tabela 3). O ano de 2005, nos dois casos, foi o que teve o maior número de publicações e o tema redes foi relevante tanto para pesquisadores nacionais como para pesquisadores internacionais. Verifica-se que, percentualmente, o termo rede foi o mais ocorrente entre as linhas estabelecidas entre os pesquisadores nacionais.

Ainda é observada na Tabela 3 a predominância de artigos que abordam a temática redes de empresas. Esse interesse na pesquisa ligada à estratégia pode refletir a preocupação de investigadores como Echols e Tsai (2005), que questionam as condições nas quais uma empresa pertencente a uma rede tem melhor desempenho. 
Tabela 3 - Número de artigos selecionados por periódico Qualis "A" de circulação nacional e internacional entre os anos de 2000 e 2005 em função do tema.

\begin{tabular}{|c|c|c|c|c|c|c|c|c|c|}
\hline & & & & & & & & & \\
\hline Âmbito & Termos & 2000 & 2001 & 2002 & 2003 & 2004 & 2005 & Total & Percentual \\
\hline & aglomeração & 2 & - & 2 & 1 & - & 3 & 8 & 20 \\
\hline & redes & 4 & 2 & 4 & 4 & 7 & 4 & 25 & 62,5 \\
\hline Nacional & aliança & 2 & 1 & - & - & - & 2 & 5 & 12,5 \\
\hline & parceria & - & 1 & - & 1 & - & - & 2 & 5 \\
\hline & Total & 8 & 4 & 6 & 6 & 7 & 9 & 40 & 100 \\
\hline & cluster & 2 & 1 & - & & & 1 & 4 & 2,9 \\
\hline & network & 24 & 5 & 5 & 8 & 16 & 24 & 82 & 58,6 \\
\hline Internacional & alliance & 16 & 2 & 6 & - & 7 & 11 & 42 & 30,0 \\
\hline & partnership & 3 & 2 & 2 & 1 & - & 4 & 12 & 8,6 \\
\hline & Total & 45 & 10 & 13 & 9 & 23 & 40 & 140 & 100 \\
\hline
\end{tabular}

Fonte: Elaborado pelos autores.

Há de se considerar que o termo pode ter distintos significados. Enquanto em Thorelli (1986) nota-se uma preocupação clara com sua aplicação em marketing; Dyer (1996) refere-se mais às linhas de produção no processo manufatureiro; e Carrão (2004) refere-se àqueles acordos que podem ser estabelecidos de forma horizontal, entre concorrentes.

Nessa mesma linha, Hoffmann, et al. (2007, p. 106) descrevem que “(...) as circunstâncias que podem ensejar sua formação [das redes] estariam ligadas à necessidade de vantagem competitiva, a todo o momento". O interesse na pesquisa talvez se paute na justificação para a criação das redes e mesmo nos seus resultados. Ainda, pode-se perceber no texto de Jarillo (1988) e Powell (1990) que sua discussão poderia até mesmo incorporar outros termos, como alianças. No entanto, o termo redes tem caráter mais amplo e, assim, maior alcance para explicar as distintas formas de relacionamentos interorganizacionais. Autores como Schommer (2001) afirmam que o termo redes foi empregado de distintas formas, até mesmo contraditórias. Talvez essa seja a razão de ele ser o mais recorrente. 


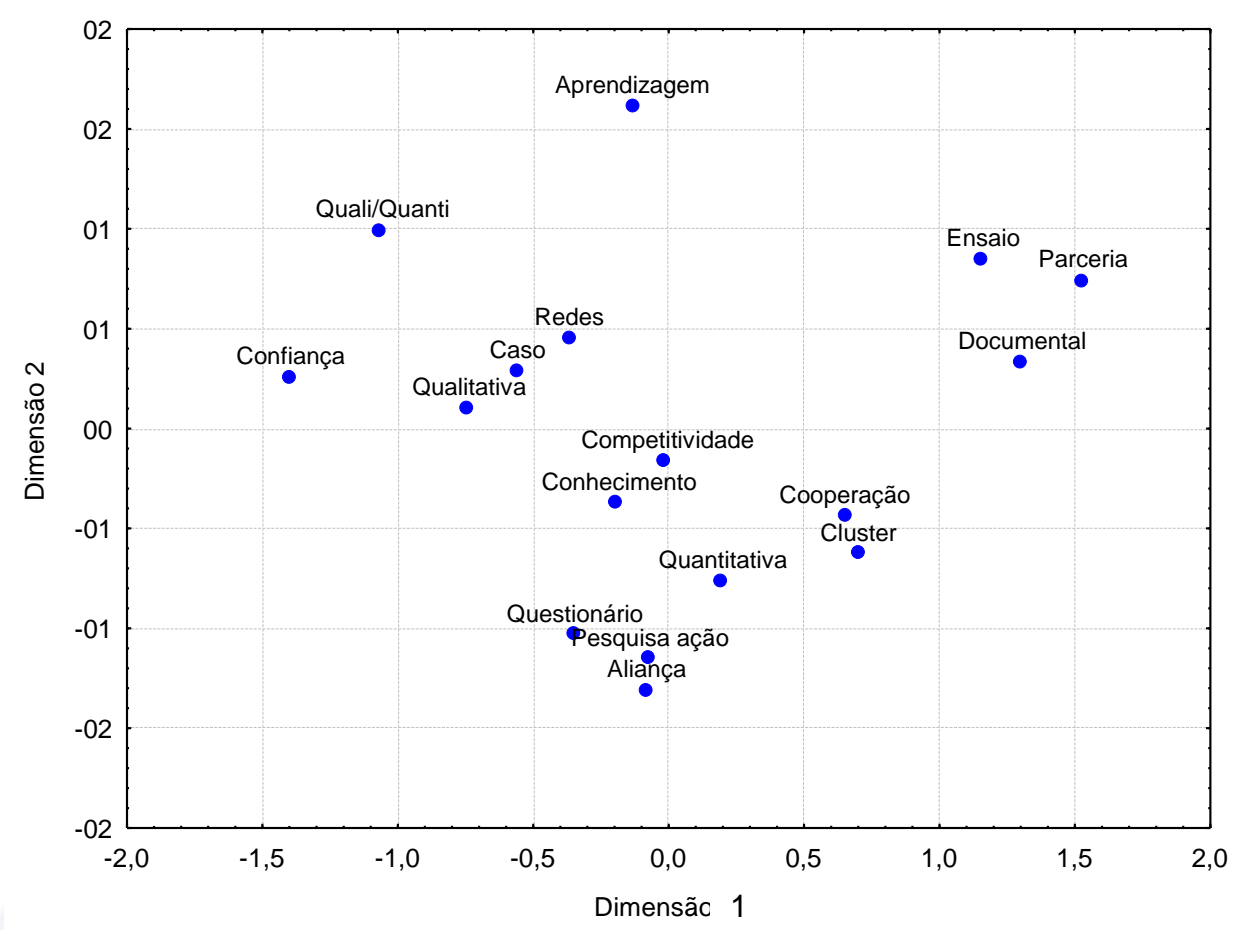

Figura 2: Mapa perceptual - termos, metodologia e estratégias de pesquisa - publicação nacional.

Fonte: Elaborado pelos autores.

A Figura 2 apresenta um mapa perceptual, criado com o intuito de mostrar associações entre essas duas dimensões: os termos e os aspectos metodológicos. Ela mostra que o termo Redes foi agrupado com o método do estudo de caso e com abordagem qualitativa. No Brasil, como já foi dito, parece haver um interesse de pesquisa mais voltado a pensar nos resultados quantificáveis das redes, como é o caso do texto de Balestrin, Vargas e Fayard (2005). O termo cluster ficou próximo de cooperação, com alguma proximidade de abordagem quantitativa de pesquisa, o que também está consoante com os primeiros trabalhos a abordar as redes no Brasil, já assinalados, como os de Amato Neto (2000); e Casarotto e Pires (1999). A pesquisa-ação associou-se ao uso de questionário como estratégia de pesquisa e ao termo aliança. Pode-se perceber que competitividade ficou praticamente equidistante de redes e cluster, e com proximidade maior com o construto conhecimento. Note-se que discutir competitividade acaba sendo um pressuposto ou justificativa dos estudos de redes, como é o caso do trabalho de Carrão (2004), ou mesmo é o escopo do próprio trabalho que trata de redes, como no trabalho de Hoffmann, Molina-Morales e Martínez-Fernandez (2008).

Um quadro bastante distinto apresenta-se na análise de correspondência das publicações internacionais (Figura 3). Alguns termos estão mais agrupados a certas metodologias. Ou seja, parecem mais evidentes algumas correspondências e não tanto outras, no caso das publicações 
internacionais. Nota-se claramente a associação do termo alianças com o construto cooperação. Desde os trabalhos de Jarillo (1988) e Thorelli (1986), em estudos internacionais, as redes são vistas mais como relações verticais, em que se minimiza a relação de força e se procura maximizar a cooperação. Isso parece justificar a associação encontrada.

Também nota-se forte conexão entre o termo parceria e o método do caso e o construto conhecimento, e entre a pesquisa documental e a abordagem qualitativa. Considerando que os periódicos consultados possuem um viés mais acentuado em estratégia, e que a pesquisa em estratégia internacionalmente possui um enfoque mais quantitativo, parece natural que esses termos, abordagem e estratégias de pesquisa apresentem alguma associação.

O que chama a atenção nesse caso é a baixa incidência de correspondência do termo cluster que ficou distante de outros termos, o que também se percebe com o termo confiança, muito embora neste último caso esteja mais próximo de aprendizagem. A abordagem quantitativa ficou equidistante de parceria e de competitividade. Com relação ao termo cluster, os estudos internacionais apresentaram um cunho mais sociológico, como é o caso de Brusco (1982); e Lorenzoni e Lipparini (1999), o que talvez explique o fato de haver pouca associação entre os termos.

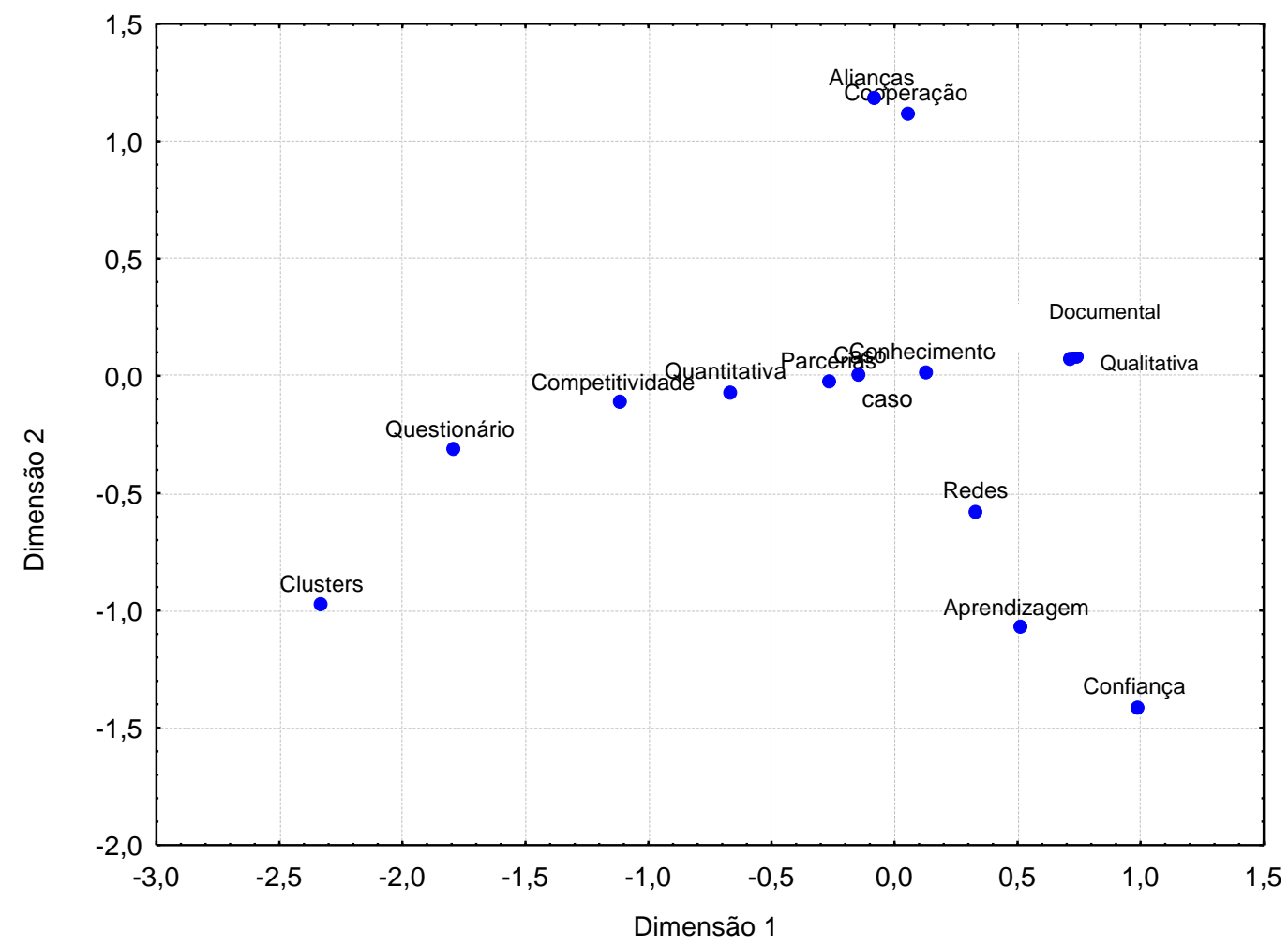

Figura 3: Mapa perceptual - termos, metodologia e estratégias de pesquisa - publicação internacional.

Fonte: Elaborado pelos autores. 
Juntando os estudos nacionais com os internacionais, o que se nota na Figura 4 é que a correspondência fica menos esclarecedora. Alguma correspondência pode ser percebida entre o construto cluster e a técnica do questionário, os construtos aliança e cooperação, e a pesquisa documental e o construto conhecimento. Isso parece indicar que a pesquisa que foi desenvolvida no Brasil diferiu daquela que foi executada no exterior no período pesquisado. Oliver e Ebers (1998) foram precursores na definição do campo de estudo de redes, embora caiba salientar o viés do campo da sociologia das fontes (revistas) que foram analisadas pelos autores. Esse trabalho parece dar uma contribuição nesse sentido, pois mostra que o campo de estudo de redes mais voltado à estratégia, no Brasil e no exterior, no período analisado, teve um desenvolvimento que pode ser descrito com uma curva "U” (Figura 1), em termos de frequência.

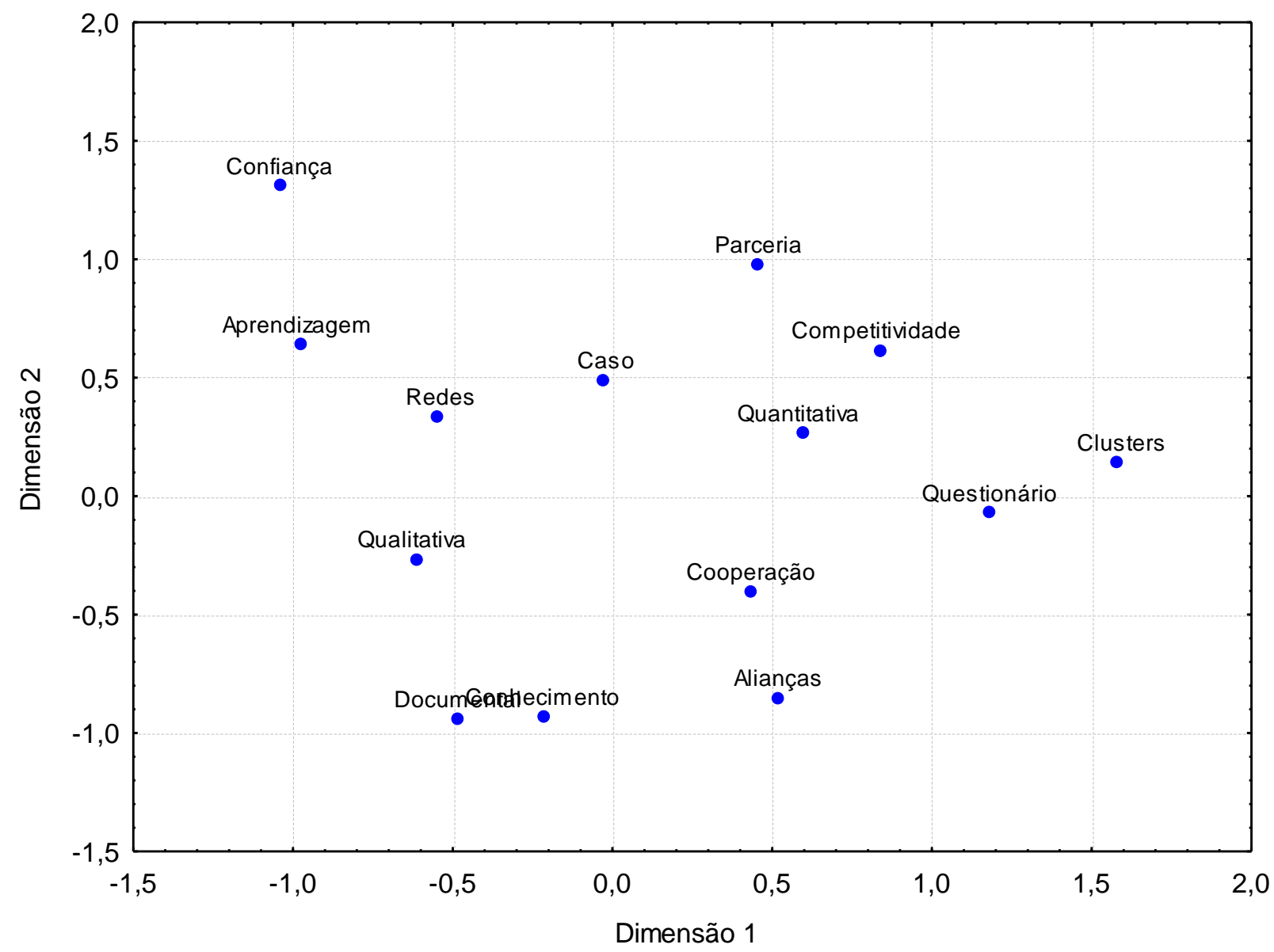

Figura 4: Mapa perceptual - termos, metodologia e estratégias de pesquisa - publicação nacional.

Fonte: Elaborado pelos autores. 
Percebe-se que no Brasil os trabalhos seguiram uma abordagem na qual a cooperação foi tratada em um contexto geográfico, em que relações interfirmas podem ser estabelecidas, como os clusters, ou aglomerações, enquanto no exterior a cooperação é entendida em relações mais estruturadas, sendo mais exploradas as questões de governança das redes como uma nova organização. Em trabalhos nacionais, as redes foram pesquisadas mais como uma abordagem qualitativa, em estudos de casos, enquanto o termo redes nos periódicos internacionais analisados ficou isolado, ficando também o termo cluster. Talvez a diferença entre esses estudos esteja nos objetos, nas características da indústria brasileira, e também que as pesquisas pioneiras no Brasil sobre cooperação/redes emergem do campo da economia, com preocupações distintas da administração e com menor foco na gestão das redes. As primeiras publicações sobre redes tratam do desenho organizacional alternativo, como fonte de vantagens competitivas (escala, aprendizagem, por exemplo), como no caso de Jarillo (1988) e Thorelli (1986), ao mesmo tempo em que discutem as economias de custos de transação (Powell, 1990; Ring \& Van De Ven, 1992). O discurso predominante sobre escala é típico das grandes empresas, como no caso do trabalho que foi desenvolvido por Dyer (1996). No Brasil, a predominância das investigações versa sobre pequenas empresas. Além disso, boa parte dos estudos foi desenvolvida usando como objeto as aglomerações territoriais (clusters), seguindo duas orientações: aquela decorrente da economia industrial, cujo expoente é Porter (1991), por um lado; e por outro, os distritos industriais, provenientes do trabalho de Marshall (1925), e mais recentemente da sociologia econômica, como nos trabalhos de Becattini (1979) e Brusco (1982). Essa fundamentação teórica distinta e também objetos de estudo diferentes podem ter influenciado as dessemelhanças.

\section{CONSIDERAÇÕES FINAIS}

Este trabalho teve como objetivo mensurar a produção de pesquisas de redes em periódicos nacionais e internacionais, indicadas pelo portal CAPES, com conceito “A” (Qualis A), até dezembro de 2005. Para isso utilizou-se a bibliometria. O trabalho indicou que no período estudado a produção sobre redes apresentou um desenvolvimento que pode ser descrito por uma curva "U", com uma frequência alta em 2000, que descendeu por volta de 2002-2003 e depois voltou a ascender em 2005. Conclui-se que o tema continua em expansão, o que pode ser justificado pelo número de edições temáticas em revistas e também em congressos na área de administração. Pode-se perceber que o Brasil acompanhou movimento internacional registrado, mas no país a descendência não foi tão alta 
quanto nas publicações do exterior, o que parece significar que há mais interesse nacionalmente sobre o tema, em comparação à produção no exterior. Isso deriva três perguntas: o crescimento das publicações nacionais se deu pelo crescimento no interesse por parte dos pesquisadores? Ou ele se deve mais ao fato de as redes terem se ampliando enquanto modelo organizacional? Ou ambas as coisas?

O termo rede apresentou maior participação dentre aqueles pesquisados, tanto no Brasil quanto no exterior. Conclui-se que a polissemia típica do termo, isto é, seu emprego para tratar de distintas configurações organizacionais, pode justificar tal resultado. Parece que o termo redes ante essa polissemia é o mais abrangente. As redes podem ser de diversos tipos, afirmam Hoffmann et al. (2007), o que indica que realidades distintas, ainda que relacionadas, podem ter se juntado neste estudo bibliométrico. A existência desses vários tipos e seu apelo junto às partes pesquisadas podem ter contribuído para que se encontrasse essa frequência.

Outra conclusão deste trabalho se refere à concentração dos estudos sobre redes em determinados periódicos. No Brasil, cerca de 30\% foram responsáveis por $45 \%$ das publicações, e, internacionalmente, 3 revistas científicas (25\%) foram responsáveis por $43 \%$ das publicações. Parece que algumas publicações foram mais receptivas ou mais procuradas que outras para a publicação de trabalhos sobre redes, o que talvez reflita o próprio interesse editorial.

As análises de correspondência efetuadas indicaram que os estudos brasileiros são distintos dos internacionais pesquisados. $\mathrm{O}$ fato de se ter objetos de estudo diferentes, no Brasil, as pequenas e médias empresas, na maioria dos casos aglomeradas territorialmente, e no exterior as grandes empresas, em um processo de desverticalização, também pode ser uma inferência cabível. Quanto ao construto cooperação, no caso do brasileiro, que está relacionado com as aglomerações territoriais (clusters), são contextos de cooperação mais horizontal. No exterior, a cooperação apresentou mais correspondência com as alianças, que são redes predominantemente diádicas, do tipo vertical.

Os resultados indicam possíveis orientações para o campo de estudos de redes no Brasil, sobretudo nos aspectos metodológicos e conceituais. Quanto à abordagem metodológica nota-se uma carência de pesquisas longitudinais, que busquem identificar a evolução do tema no contexto das organizações brasileiras. No que se refere aos aspectos conceituais, os trabalhos estão fortemente ancorados à economia industrial, carecendo, ainda, de investigações com o escopo de analisar as dimensões da gestão das redes e de aspectos relacionados aos limites desse modelo para a competitividade das organizações. Para o campo da estratégia, almeja-se alimentar novas discussões inerentes à dimensão da cooperação, como uma opção estratégica para o alcance de competitividade das organizações contemporâneas.

Revista de Administração e Inovação, São Paulo, v. 8, n. 2, p.29-54, abr./jun. 2011 
Este trabalho está limitado ao período de análise. Como o ano de 2005 apontou para um crescimento, a necessidade de ampliar esta pesquisa ao menos para 2008 é premente. Outro limite se refere ao acesso às bases de dados, o que indica que com outras bases será possível incluir novos periódicos. Esses são caminhos para a continuidade deste trabalho.

\section{REFERÊNCIAS}

Amato Neto, J. (2005). Redes dinâmicas de cooperação e organizações virtuais. In J. Amato Neto (Org.), Redes entre organizações: domínio do conhecimento e da eficácia operacional (pp. 1738). São Paulo: Atlas.

Amato Neto, J. (2000). Redes de cooperação produtiva e cluster regionais: oportunidades para as pequenas e médias empresas. São Paulo: Atlas.

Balestrin, A., Vargas, L. M., \& Fayard, P. (2005). O efeito rede em pólos de inovação: um estudo comparativo. Revista de Administração, 40(2), 159-171.

Balestrin, A., Verschoore, J. (2008). Redes de cooperação empresarial: estratégias de gestão na nova economia. Porto Alegre: Bookman.

Balestrin, A., Verschoore, J. R., \& Reyes Junior, E. (2010). O campo de estudo sobre redes de cooperação interorganizacional no brasil. Revista de Administração Contemporânea, 14(3), 458477.

Becattini, G. (1979). Dal settore industriale al distretto industriale. alcune considerazioni sull' unità di indagine dell'economia industriale. Rivista di Economia e Política Industriale, 1, 7-21.

Begnis, H. S. M., Pedrozo, E. A., \& Estivalete, V. F. B. (2005). Cooperação enquanto estratégia segundo diferentes perspectivas teóricas [CD-ROM]. Anais do Encontro da Associação Nacional de Pós-Graduação e Pesquisa em Administração, 29. Rio de Janeiro: ANPAD, 2005.

Brusco, S. (1982). The Emilian model: productive decentralization and social integration. Cambridge Journal of Economics, 6(1), 167-184. 
Carrão, A. M. R. (2004). Cooperação entre empresas de pequeno porte em pólos industriais: um estudo comparativo. Revista de Administração, 39(2), 198-195.

Carter, C. R., Leuschner, R., \& Rogers, D. S. (2007). A social network analysis of the Journal of Supply Chain Management: knowledge generation, knowledge diffusion and thought leadership. Journal of Supply Chain Management, 43(2), 15-28.

Casarotto Filho, N., \& Pires, L. H. (1999). Redes de pequenas e médias empresas e desenvolvimento local: estratégias para a conquista da competitividade global com base na experiência italiana. São Paulo: Atlas.

Cassiolato, J. E., \& Szapiro, M. (2003). Alguns indicadores de inovação no Brasil: os dados da PINTEC e a importação de tecnologia. In J. E. Cassiolato, \& M. M. H. Lastres (Org), Novas políticas de inovação lições para o Brasil. Rio de Janeiro: IE/URFJ. (Mimeografado).

Castells, M. (1999). A sociedade em rede (2 ed.) São Paulo: Paz e Terra.

Chan, B. L., Milani Filho, M. A. F., \& Martins, G. A. (2007). Utilização da análise de correspondência para uma abordagem bibliométrica [CD-ROM]. Anais do Encontro da Associação Nacional de Pós-Graduação e Pesquisa em Administração, 31. Rio de Janeiro: ANPAD.

Chung, K. H., \& Cox, R. A. K. (1990). Patterns of productivity in the finance literature: a study of the bibliometric distributions. Journal of Finance, 45(1), 301-309.

Chung, K. H., Pak. H. S., \& Cox. R. A. K. (1992). Patterns of research output in the accounting literature: a study of bibliometric distributions. Abacus, 2(2), 168-185.

Coyne, K. P. (1986). Sustainable competitive advantage - what it is, what it isn't. Business Horizons, 29(1), 54-61.

Culnan, M. J. (1986). The intellectual development of management information systems, 1972-1982: a co-citation analysis. Management Science, 32(2), 156-172.

Dyer, J. H. (1996). Specialized supplier networks as a source of competitive advantage: evidence from the auto industry. Strategic Management Journal, 17(4), 271-291. 
Echols, A., \& Tsai, W. (2005). Niche and performance: the moderating role of network embeddedness. Strategic Management Journal, 26, 219-238.

Frey, K. (2003). Governança urbana e redes sociais: o potencial das novas tecnologias da informação e comunicação [CD-ROM]. Anais do Encontro da Associação Nacional de Pós-Graduação e Pesquisa em Administração, 27. Rio de Janeiro: ANPAD.

Gallon, A. V., \& Cunha, C. J. C. A. (2007). Investigando os estudos etnográficos publicados nos anais do Enanpad: uma análise de 2000 a 2006 [CD-ROM]. Anais do Encontro de Ensino e Pesquisa em Administração e Contabilidade, 1. Rio de Janeiro: ANPAD.

Gallon, A. V., Souza, F. C., Rover, S., \& Ensslin, S. R. (2007). Estratégias metodológicas da produção científica em capital intelectual: uma análise de 2000 a 2006 [CD-ROM]. Anais do Encontro de Ensino e Pesquisa em Administração e Contabilidade, 1. Rio de Janeiro: ANPAD.

Guedes, V. L. S., \& Borschiver, S. (2007). Bibliometria: uma ferramenta estatística para gestão da informação e do conhecimento, em sistemas de informação, de comunicação e de avaliação científica e tecnológica. Recuperado em 29 fevereiro, 2007 de http://dici.ibict.br/archive/00000508/01/VaniaLSGuedes.pdf.

Hall, C. M. (2001). Planejamento turístico: políticas, processos e relacionamentos. São Paulo: Contexto.

Hoffman, D. L., \& Holbrook, M. B. (1993). The intellectual structure of consumer research: a bibliometric study of author cocitations in the first 15 years of the Journal of Consumer Research. Journal of Consumer Research, 19(4), 505-517.

Hoffmann, V. E., Molina-Morales, F. X., \& Martínez-Fernandez, M. T. (2008). Competitividade na indústria do vestuário: uma avaliação a partir da perspectiva das redes de empresas aglomeradas territorialmente. Revista Eletrônica de Administração, 14(2), 1-29.

Hoffmann, V. E., Molina-Morales, X. F., \& Martinez-Fernádez, M. T. (2007). Redes de empresas: proposta de uma tipologia para classificação aplicada na indústria de cerâmica de revestimento. Revista de Administração Contemporânea, 11(n.spec. 1), 103-127.

Jarillo, J. C. (1988). On strategic networks. Strategic Management Journal, 9(1), 31-41. 
Knorringa, P., \& Meyer-Stamer, J. (1999). New dimensions in local enterprise co-operation and development: from clusters to industrial districts. In W. Suzigan (Coord.), Clusters e sistemas locais de inovação: estudos de casos e avaliação da região de Campinas (pp. 89-115). Campinas: Unicamp.

Kostoff, R. N. et al. (2007). Clustering methodologies for identifying country core competencies. Journal of Information Science, 3(1), 21-40.

Lewis, J. (1992). Alianças estratégicas: estruturando e administrando parcerias para o aumento da lucratividade. São Paulo: Pioneira.

Lorenzoni, G., \& Lipparini, A. (1999). The leveraging of interfirm relationships as a distintive organizational capability: a longitudinal study. Strategic Management Journal, 20(4), 317-338.

Macedo, M. A. S., Nova, S. P. C. C., \& Almeida, K. (2007). Mapeamento e análise bibliométrica da utilização da análise envoltória de dados (DEA) em estudos das áreas de contabilidade e administração [CD-ROM]. Anais do Encontro da Associação Nacional de Pós-Graduação e Pesquisa em Administração, 31. Rio de Janeiro: ANPAD.

Magalhães, F. A. C. (2006). Construção do saber no programa de doutorado [CD-ROM]. Anais do Encontro da Associação Nacional de Pós-Graduação e Pesquisa em Administração, 30. Rio de Janeiro: ANPAD.

Mahon, J. F., \& Andrews, P. N. (1987). Social issues in management literature: a preliminary citation analysis. Academy of Management Best Papers Proceedings, 344-348.

Marshall, A. (1925). Principles of economics (8 ed.). London: Macmillan.

McMillan, G. S., \& Casey, D. L. (2007). Research note: identifying the invisible colleges of the British journal of industrial relations: a bibliometric and social network approach. British Journal of Industrial Relations, 45(4), 815-828.

McMillan, G. S., \& Hamilton III, R. D. (2000). Using bibliometrics to measure firm knowledge: an analysis of the us pharmaceutical industry. Technology Analysis \& Strategic Management, 12(4), 465-475. 
Mendonça Neto, O. R., Riccio, E. L., \& Sakata, M. C. G. (2006). Paradigmas de pesquisa em contabilidade no Brasil. [CD-ROM]. Anais do Encontro da Associação Nacional de PósGraduação e Pesquisa em Administração, 30. Rio de Janeiro: ANPAD.

Miles, R. E., \& Snow, C. C. (1986). Organizations: new concepts for new forms. California Management Review, 28(3), 62-73.

Moretti, S. L. A., \& Figueiredo, J. C. B. (2007). Análise bibliométrica da produção sobre responsabilidade social das empresas no enanpad: evidências de um discurso monológico [CDROM]. Anais do Encontro da Associação Nacional de Pós-Graduação e Pesquisa em Administração, 31. Rio de Janeiro: ANPAD.

Nakano, D. N. (2005). Fluxos de conhecimento em redes interorganizacionais: conceitos e fatores de influência. In J. Amato Neto (Org.), Redes entre organizações (pp. 54-67). São Paulo: Atlas.

O'leary, D. E. (2007). The most cited papers. Human Systems Management, 26(3), 153-156.

Oliver, A. L., \& Ebers, M. (1998). Networking network studies: an analysis of conceptual configurations in the study of inter-organizational relationships. Organization Studies, 19(4), 549583.

Pasadeos, Y. (1985). A bibliometric study of advertising citations. Journal of Advertising, 14(4), 5268.

Pitassi, C., \& Macedo-Soares, T. D. L. V. A. (2003). Redes estratégicas: fatores críticos de sucesso. Revista de Administração Contemporânea, 7,(n.spe), 75-99.

Porter, M. E. (1991). Towards a dynamic theory of strategy. Strategic Management Journal, 12, 95117.

Powell, W. W. (1990). Neither market nor hierarchy: networks forms of organization. Research of Organizational Behaviour, 12, 295-336.

Puffal, D. P., \& Tondolo, V. A. G. (2008). A evolução do campo de estudo de redes de interorganizacionais: uma análise de publicações internacionais das relações entre empresas [CDROM]. Anais do Encontro de Estudos Organizacionais, 5. Rio de Janeiro: ANPAD. 
Ring, P. S., \& Van De Ven, A. H. (1992). Structuring cooperative relationships between organizations. Strategic Management Journal, 13, 483-498.

Rossoni, L., Hocayen-da-Silva, A. J., \& Ferreira Júnior, I. (2008). Aspectos estruturais da cooperação entre pesquisadores no campo de administração pública e gestão social: análise das redes entre instituições no Brasil. Revista de Admnistração Pública, 42(6), 1041-1067.

Schommer, P. C. (2001). Gestão de organizações sem fins lucrativos: algumas questões sobre as "especificidades" do campo e o perfil dos gestores (pp. 273-278). Anais do Congresso Nacional das APAES, 20. Brasília: Federação Nacional das APAEs.

Siqueira, M. M. (2000). Redes sociais na gestão de serviços urbanos. Revista de Administração Pública, 34(6), 98-179.

Teixeira, F., \& Guerra, O. (2002). Redes de aprendizado em sistemas complexos de produção. Revista de Administração de Empresas, 42(4), 93-105.

Thorelli, H. B. Networks: between markets and hierarchies. Strategic Management Journal, 7(1), 3751.

Verschoore Filho, J. R. S. (2003). O programa redes de cooperação: uma análise dos instrumentos de administração pública para o desenvolvimento sócio-econômico (pp. 28-31). Anais do Congreso Internacional del CLAD sobre la Reforma del Estado y de la Administración Pública, 8. Panamá: CLAD.

Williamson, O. E. (1991). Comparative economic organization: the analysis of discrete structural alternatives. Administrative Science Quarterly, 36(2), 269-296. 


\title{
ANALYSIS OF PRODUCTION IN THE FIELD OF SCIENTIFIC STUDY OF NETWORKS IN NATIONAL AND INTERNATIONAL JOURNALS
}

\begin{abstract}
This study measures the production of research networks, through the final product of research scientific publications. Several theoretical approaches to networks offer the potential for dialogue among the various branches of the social sciences. Contemporary authors say that companies in search of competitiveness have found networks to be a strategic alternative. Since the first studies in the 1980s, consciousness of these issues has risen in Brazil and around the world. Expanding in a nonlinear way, the state of the art has expanded, but without giving a unified response on simple matters, like the concept of networks. According to this study scope, we chose the methodology of bibliometrics, applied in national and international journals (Brazilian Scientific Journal Classification - Qualis A), publications which focused on strategy, for the period from 2000 to 2005, in the area of Business Administration. The results indicate the prevalence of the term networks in national and international studies; about $30 \%$ of the surveyed journals published approximately $45 \%$ of their articles on this subject; the number of publications in 2000 was higher than in 2002 -- 2003, but it increased again in 2005; and finally, there are differences between Brazilian studies and international ones.
\end{abstract}

Keywords: Networks; Inter-organizational relationship; Clusters; Bibliometrics.

Data do recebimento do artigo: 15/12/2011

Data do aceite de publicação: 04/03/2011 